\title{
Cryo Imaging of Life Science and Soft matter Samples by a State of the Art $120 \mathrm{kV}$ EFTEM
}

\author{
M. Thaler*, S. A. Hiller*, C. Dietl*, V. Seybold* and G. Benner* \\ * Carl Zeiss NTS GmbH, D-73447 Oberkochen, Germany
}

Cryo Transmission Electron Microscopy (cryoTEM) of frozen hydrated samples is a modern approach which has been established to go far beyond the static ultrastructural imaging of tissue to reveal structural and functional information of native structures in biological and soft-matter systems (Fig 1). Cryo TEM is a powerful technique for the visualisation of delicate and dynamic structures without the introduction of artefacts due to conventional staining or drying procedures.

During the last years the method has significantly contributed to the understanding of divers and complex substances in aqueous solutions. In particular, many studies using CryoTEM on liposomes (Fig. 2) used as carrier for drugs have been presented ([1], [2], [3], [4]). CryoTEM supports studies of structural and functional properties of bio-inspired surfactants for controlled fabrication of sophisticated nano-carriers and gets growing importance in pharmaceutical applications to exploit lipids as models in nanotechnology. It allows investigations on structural transitions of aggregates and substances e.g. DLPA micellar solutions upon thermal treatment ([5], [6]) which can help molecular design of functional surfactants and increase knowledge about disease-related peptides [7].

In-column energy filter transmission electron microscopes (EFTEM`s) feature fundamentally advantages for cryo investigations which greatly surpass the performance of modern conventional TEM`s and clearly extend their limits [8], [9]. This presentation will cover the benefits of EFTEM`s for cryo imaging. The newly introduced EFTEM Libra $120^{\circledR}$ PLUS of Carl Zeiss is available in a dedicated cryo configuration. Dedicated Low Dose Plug-ins for image processing systems are available which allow a complete and easy to use automation of low dose experiments taking full advantage of the flexibility of the Köhler illumination system [10] to reduce beam damage of a frozen sample to the absolute minimum. All the basic advantages of the Libra $120^{\circledR}$ EFTEM series become even more effective due to a new vacuum system. A constitutional redesign of the column hardware now offers significantly improved partial pressure rates around the specimen area which allow prolonged cryo work with negligible ice grow rate $<1 \mathrm{~nm} / \mathrm{h}$. The new Libra $120^{\circledR}$ PLUS vacuum systems are fieldupgradeable, permitting to start with the basic version minimizing ownership costs but safeguarding future applications that can not be seen yet.

The redesigned electron optical column, completely dry vacuum system and the integrated OMEGA energy filter as an imaging element combined with most flexible illumination concept (Köhler) available for TEM`s outlines this instrument to an easy workhouse for everyday's cryo applications in a wide application range.

The benefits of EFTEM using the L120 ${ }^{\circledR}$ PLUS for cryo applications will be explained and some highlights of results will be presented. 
[3] L. Ickenstein, M-C. Sandström, L-D. Mayer, K. Edwards, 2006: Effects of phospholipid hydrolysis on the aggregate structure in nDPPC/DSPE-PEG2000 liposome preparations after gel to liquid crystalline phase transition, Biochimica et Biophysica Acta 1758 (2006) $171-180$

[4] A. Lundquist, P. Wessman, AR. Rennie, K. Edwards, 2008: Melittin-Lipid interaction: A comparative study using liposomes, micelles and bilayer disks, Biochimica et Biophysica Acta 1778 (2008) 2210-2216

[5] FB. Bombelli, D. Berti, S. Milani, M. Lagi, P. Barbaro, G. Karlsson, A. Brandt, P. Baglioni, 2008: Collective headgroup conformational transition in twisted micellar Superstructures, Soft Matter, 2008, 4, 1102-1113

[6] A-S. Johansson, A. Garlind, F. Berglind-Dehlin, G. Karlsson, K. Edwards, P Gellerfors, F. Ekholm-Pettersson, J. Palmblad, L. Lannfelt, 2007: Docosahexaenoic acid stabilizes soluble amyloid-b protofibrils and sustains amyloid-b-induced neurotoxicity in vitro, FEBS Journal 274 (2007) 990-1000

[7] A-S. Johansson, F. Berglind-Dehlin, G. Karlsson, K. Edwards, P. Gellerfors, L. Lannfelt, 2006: Physiochemical characterization of the Alzheimer's disease-related peptides Ab1-42Arctic and Ab1-42wt, FEBS Journal 273 (2006) 2618-2630

[8] J. Zhu, PA. Penczek, R. Schröder, J. Frank, 1997: Three-dimensional reconstruction with contrast transfer function correction from energy-filtered cryoelectron micrographs: procedure and application to the 70S Escherichia coli ribosome. J Struct Biol. 1997 Apr; 118(3):197-219.

[9] R-R. Schroder, W. Hofmann, J.-F., Menetret, 1991: Zero-loss energy filtering as improved imaging mode in cryoelectronmicroscopy of frozen hydrated specimens. Journal of structural biology 105 (1991), 28-34

[10] G. Benner, W. Probst, 1994: Köhler Illumination in the TEM: fundamentals and advantages, Journal of Microscopy 174-3 (1994), 133142


\section{Fig 1}

Cryo-TEM micrographs of block-copolymers, images are zero-loss filtered. Images courtesy of M. Drechsler, E. Egbali \& H. Hofmann, Univ. Bayreuth, Germany

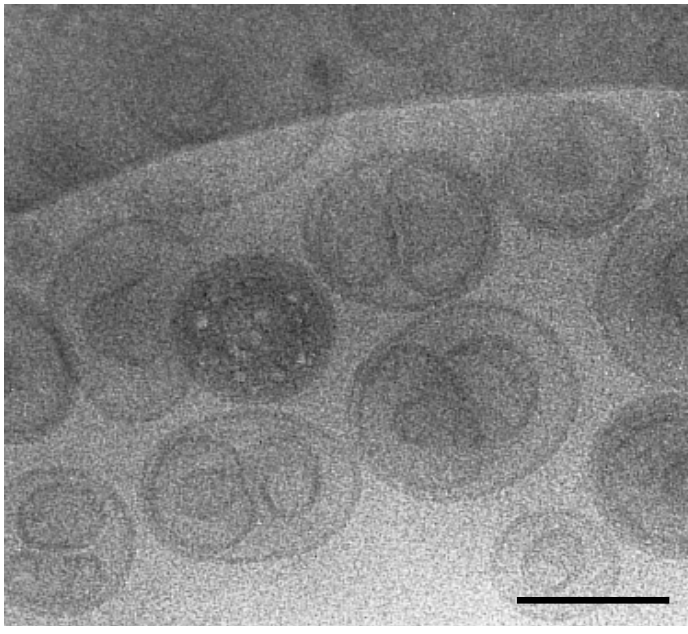

Unfiltered image

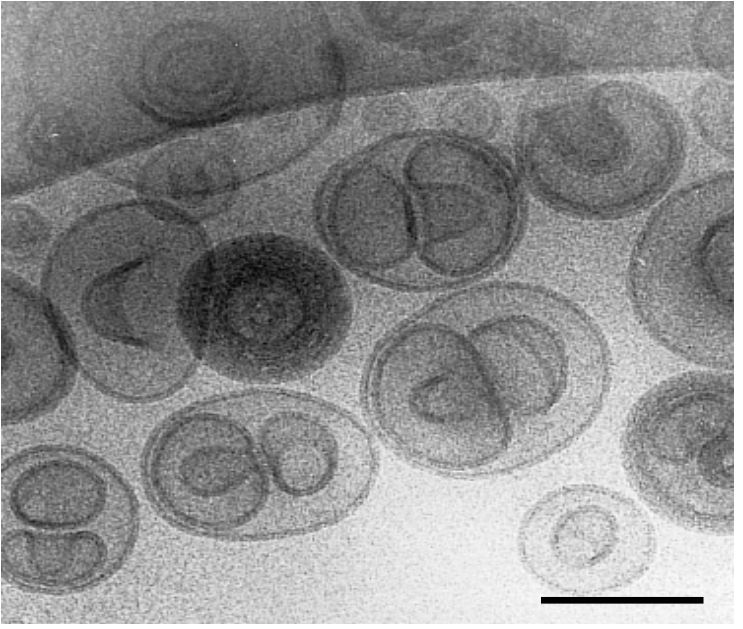

Filtered image

Fig. 2

Frozen hydrated liposomes: Comparison between Conventional and Energy filtered TEM imaging. Scale bar $=100 \mathrm{~nm}$. Comparison between unfiltered (left) and Zero loss filtered image (right) shows higher contrast, less noise and better resolution for the filtered low dose image 